\title{
Calcitriol analog ZK191784 ameliorates acute and chronic dextran sodium sulfate-induced colitis by modulation of intestinal dendritic cell numbers and phenotype
}

\author{
Ulrike G Strauch, Florian Obermeier, Nicole Grunwald, Nadja Dunger, Heiko C Rath, Jürgen Schölmerich, \\ A Steinmeyer, U Zügel, HH Herfarth
}

\begin{abstract}
Ulrike G Strauch, Florian Obermeier, Nicole Grunwald, Nadja Dunger, Heiko C Rath, Jürgen Schölmerich, HH Herfarth, Department of Internal Medicine I, University of Regensburg, Regensburg D-93053, Germany

A Steinmeyer, Medicinal Chemistry, Schering AG, Berlin, Germany

U Zügel, Research Business Area Dermatology, Schering AG, Berlin, Germany

Supported by Grants from the German Research Foundation DFG (UGS, HCR), as well as research grants from the University of Regensburg, Germany, as part of the ReForMprogram (UGS, FO)

Correspondence to: Ulrike G Strauch, Department of Internal Medicine I, University of Regensburg, Regensburg D-93053, Germany. ulrike.strauch@klinik.uni-regensburg.de

Telephone: +49-941-9447001 Fax: +49-941-9447002

Received: June 24, 2007 Revised: September 2, 2007
\end{abstract}

\begin{abstract}
AIM: To investigate the effects of ZK1916784, a low calcemic analog of calcitriol on intestinal inflammation.

METHODS: Acute and chronic colitis was induced by dextran sodium sulfate (DSS) according to standard procedures. Mice were treated intraperitoneally with ZK1916784 or placebo and colonic inflammation was evaluated. Cytokine production by mesenterial lymph node (MLN) cells was measured by ELISA. Immunohistochemistry was performed to detect intestinal dendritic cells (DCs) within the colonic tissue, and the effect of the calcitriol analog on DCs was investigated.
\end{abstract}

RESULTS: Treatment with ZK191784 resulted in significant amelioration of disease with a reduced histological score in acute and chronic intestinal inflammation. In animals with acute DSS colitis, downregulation of colonic inflammation was associated with a dramatic reduction in the secretion of the proinflammatory cytokine interferon (IFN) $-\gamma$ and a significant increase in intereleukin (IL)-10 by MLN cells. Similarly, in chronic colitis, IL-10 expression in colonic tissue increased 1.4-fold when mice were treated with ZK191784, whereas expression of the Th1-specific transcription factor T-beta decreased by $81.6 \%$. Lower numbers of infiltrating activated CD11 $\mathrm{C}^{+} \mathrm{DCs}$ were found in the colon in ZK191784-treated mice with acute DSS colitis, and secretion of proinflammatory cytokines by primary mucosal DCs was inhibited in the presence of the calcitriol analog.

CONCLUSION: The calcitriol analog ZK191784 demonstrated significant anti-inflammatory properties in experimental colitis that were at least partially mediated by the immunosuppressive effects of the derivate on mucosal DCs.

\section{(c) 2007 WJG. All rights reserved.}

Key words: Dextran sodium sulfate colitis; Calcitriol; Colonic inflammation; Dendritic cells

Strauch UG, Obermeier F, Grunwald N, Dunger N, Rath HC, Schölmerich J, Steinmeyer A, Zügel U, Herfarth HH. Calcitriol analog ZK191784 ameliorates acute and chronic dextran sodium sulfate-induced colitis by modulation of intestinal dendritic cells numbers and phenotype. World J Gastroenterol 2007; 13(48): 6529-6537

http://www.wjgnet.com/1007-9327/13/6529.asp

\section{INTRODUCTION}

Inflammatory bowel disease (IBD) is a chronic immune-mediated disease of the gastrointestinal tract of still unknown etiology, most commonly involving inflammation of the terminal ileum and colon. The disease is characterized by deregulated immune responses, and in particular, $\mathrm{CD}^{+} \mathrm{T}$ cells that produce large amounts of proinflammatory cytokines [such as interleukin (IL)-2, interferon (IFN) $-\gamma$ and tumor necrosis factor (TNF- $\alpha$ ) have been shown to play a central role ${ }^{[1]}$. Besides genetic factors that are thought to predispose individuals to develop IBD ${ }^{[2]}$, the environment seems to contribute to the disease $\mathrm{s}^{[3,4]}$. There is reason to believe that vitamin $\mathrm{D}$ may be an environmental factor that affects IBD, as vitamin $\mathrm{D}$ deficiency has been linked to ulcerative colitis and Crohn's disease even when the disease is in remission ${ }^{[5,6]}$. Additionally, vitamin $\mathrm{D}$ deficiency has been shown to accelerate the development of IBD symptoms among IL-10 knockout mice that develop spontaneous enterocolitis ${ }^{[7]}$. Also, calcitriol treatment reduces the ability of IL-10 knockout mice to produce and to respond to 
TNF- $\alpha^{[8]}$, and it has recently been shown that application of a calcitriol analog reduces intestinal inflammation in a 2,4,6-trinitrobenzene sulfonic acid colitis model ${ }^{[9]}$.

Calcitriol is the activated form of vitamin $\mathrm{D} 3$ that binds to a nuclear receptor protein. In the $\mathrm{CD} 4^{+} \mathrm{CD} 45 \mathrm{RB}^{\text {high }}$ transfer model of colitis, in which T- and B-cell deficient recipient mice develop IBD symptoms when injected with $\mathrm{CD}^{+} \mathrm{CD} 45 \mathrm{RB}^{\text {high }} \mathrm{T}$ cells, lymphocytes from vitamin $\mathrm{D}$ receptor (VDR) knockout mice increase colitis severity in recipient mice compared to similar cells from wild-type animals ${ }^{[10]}$. Also, VDR knockout mice develop a more severe colitis in response to dextran sodium sulfate (DSS) treatment than wild-type mice, which can be decreased in severity by dietary or rectally administered calcitriol ${ }^{[1]}$. To underline the importance of calcitriol in IBD, the VDR gene has been mapped to a region on chromosome 12 that has been shown to be linked to susceptibility for Crohn's disease, which suggests an influence of calcitriol on the intestinal immune system ${ }^{[12]}$.

The immunoregulatory effects of the cellular receptor for calcitriol are complex and not yet finally understood. However, as the VDR was discovered in resting and activated lymphocytes ${ }^{[13]}$, it has been suggested that calcitriol might be involved in immunoregulation. In particular, $\mathrm{CD} 4^{+} \mathrm{T}$ cells that are known to play a major role in chronic colitis express VDR, and are among the identified targets of activated vitamin $\mathrm{D}^{[14,15]}$. Indeed, calcitriol is able to inhibit $\mathrm{T}$-cell proliferation and secretion of different proinflammatory cytokines in vitro ${ }^{[13,16]}$, and to suppress the development of several experimental models of chronic inflammatory diseases, such as rheumatoid arthritis, experimental autoimmune encephalomyelitis and diabetes in vivo ${ }^{[7,10,17-19]}$. Also, the presence of calcitriol significantly inhibits cell proliferation of $\mathrm{T}$ lymphocytes obtained from patients with ulcerative colitis ${ }^{[20,21]}$. In mice treated with calcitriol, increased production of TGF- $\beta 1$ and IL-4 was observed ${ }^{[2]}$, cytokines that are associated with the inhibition of $\mathrm{T}$ cell effector function in a murine model of colitis $^{[23]}$. Furthermore, calcitriol has been found to inhibit dendritic-cell (DC) maturation, which leads to reduced alloreactive capacity ${ }^{[24]}$ and enhanced generation of regulatory $\mathrm{CD} 4^{+} \mathrm{CD} 25^{+} \mathrm{T}$ cells ${ }^{[25]}$.

Calcitriol selectively regulates the immune response without compromising host ability to fight infection ${ }^{[17]}$, which makes it an attractive target compared to standard immunosuppressive medication in patients with IBD. However, calcitriol has clear dose-limiting hypercalcemic effects that interfere with its systemic clinical use due to strong influence on calcium homeostasis and the risk of associated side effects. Recently, ZK191784, a new low-calcemic calcitriol derivative, has been identified that inhibits lymphocyte proliferation and suppresses secretion of proinflammatory cytokines by monocytes ${ }^{[2]}$. The compound has shown immunosuppressive activity in a murine contact hypersensitivity model when given systemically at concentrations that do not cause hypercalcemic effects, thereby suggesting a possible therapeutic application as an immunosuppressive agent ${ }^{[26]}$.

The aim of our study was to investigate whether this modified calcitriol analog might have potential therapeutic value in the treatment of acute and chronic intestinal inflammation, using the DSS model of colitis.

\section{MATERIALS AND METHODS}

\section{Mice}

Female Balb/c mice were obtained from Charles River (Germany) and were used for the experiments at 6-8 wk of age and 20-22 g body weight. Animals obtained food and water ad libitum. The local Institutional Review Board approved the animal studies.

\section{Reagents and antibodies}

The calcitriol analog ZK191784 was synthesized as previously described ${ }^{[26]}$, dissolved in ethanol at a concentration of $1 \times 10^{-2} \mathrm{~mol} / \mathrm{L}$ and kept at $-20^{\circ} \mathrm{C}$ until use. The following antibodies were used in the study (all purchased from BD Pharmingen, Heidelberg, Germany): anti-CD3, anti-CD11c, anti-CD28, anti-CD16/CD32, antiMHC- II, anti-CD40, anti-CD80, and anti-CD86.

\section{Induction and treatment of DSS colitis}

DSS (molecular mass 40000) was purchased from ICN (Eschwege, Germany). Acute colitis was induced by feeding 3\% DSS over $7 \mathrm{~d}^{[27]}$. Treatment with ZK191784 $(100 \mu \mathrm{g} / \mathrm{kg}$ per day orally) or vehicle was either started on day -3 before DSS administration and maintained through to $\mathrm{d} 7$ ("pretreatment"), or started on the first day of DSS application through d 7 ("treatment"). Mice were sacrificed on d 8. For induction of chronic colitis, mice received four cycles of DSS treatment. Each cycle was followed by a period of $10 \mathrm{~d}$ water without DSS. Treatment was performed before the first and before the third cycle of DSS for $7 \mathrm{~d}$ each $(100 \mu \mathrm{g} / \mathrm{kg}$ per day orally). The animals were killed on $\mathrm{d} 8$ after completion of the fourth cycle.

\section{Histological examination}

From the distal third of the colon, $1 \mathrm{~cm}$ of tissue was harvested from each animal, embedded in paraffin, stained with hematoxylin/eosin after sectioning, and used for histological analysis. To quantify the tissue damage, a scoring system was used as described previously ${ }^{[27]}$. Thereby, three sections, each obtained at a distance of $100 \mu \mathrm{m}$ were evaluated. Mice were scored individually, each score representing the mean of three sections. Two independent investigators, blinded to the treatment group, performed histological examination. The total histological score represented the sum of the epithelium and infiltration score, and ranged from 0 to $8^{[27]}$.

\section{Cytokine ELISA of mesenterial lymph node (MLN) cells}

MLN cells (pooled from each group of mice) were collected in cold cell-culture medium [RPMI-1640, 10\% fetal calf serum (FCS), $100 \mathrm{U} / \mathrm{mL}$ penicillin and $100 \mu \mathrm{g} /$ $\mathrm{mL}$ streptomycin; GIBCO-BRL, Eggenstein, Germany] and $\beta$-mercaptoethanol $\left(3 \times 10^{-5} \mathrm{~mol} / \mathrm{L}\right.$; Sigma $)$. Tissues were mechanically disrupted and the cell suspensions were filtered through a cell strainer $(70 \mu \mathrm{m})$. Tissueculture plates were coated in part with anti-CD3 (2.5 $\mu \mathrm{g} /$ well), and $2 \times 10^{5}$ cells/well were incubated in $200 \mu \mathrm{L}$ 
complete medium containing $10 \mathrm{U} / \mathrm{mL}$ IL-2 (Proleukin; Chiron) and in part with soluble anti-CD28 $(1 \mu \mathrm{g} / \mathrm{mL})$ for $24 \mathrm{~h}$. Cytokine levels were measured in the supernatant by ELISA (all from Endogene, Woburne, MA, USA), according to the manufacturer's instructions.

\section{Quantitative RT-PCR (light cycler)}

RNA was extracted from colonic tissue using the RNeasy kit (Qiagen, Hilden, Germany) and transcribed (Promega, Mannheim, Germany). Quantification of cytokine mRNA was performed using a light cycler (Roche, Molecular Systems, Mannheim, Germany). The following primer pair was used for amplification of T-beta: 5'-AGGCTGCCTGCAGTGCT'TC-3'and 5'-CTCGC CTGGTGAAATGTGC-3', annealing temperature at $62^{\circ} \mathrm{C}$, and $3 \mathrm{mmol} / \mathrm{L} \mathrm{MgCl}$. For IL-10 amplification, the following primer pair was used: 5'-TCCT'TAATGCAG GACTTTAAGGGTTACTTG-3'and 5'-GACACCTTG GTCTTGGAGCTTATTAAAATC-3', annealing temperature $62^{\circ} \mathrm{C}$, and $3 \mathrm{mmol} / \mathrm{L} \mathrm{MgCl}$. For standardization, $\beta$-actin was amplified using the following primer pair: 5'-TGGAATCCTGTGGCATCCATGAAAC$3^{\prime}$ and 5'-TAAAACGCAGCTCAGTAACAGTCCG-3'.

\section{Generation of bone-marrow-derived DCs and isolation of CD11c ${ }^{+}$primary DCs}

Bone-marrow-derived DCs (BM-DCs) were generated as described previously ${ }^{[28]}$. Briefly, bone marrow was flushed from femurs and tibiae of mice, and cells cultured for $10 \mathrm{~d}$ with PRMI (RPMI, 10\% FCS, 1\% penicillin/streptomycin, $1 \%$ l-glutamine, and $0.1 \% 2$-mercaptoethanol) containing $200 \mathrm{U} / \mathrm{mL}$ granulocyte-macrophage colony-stimulating factor (GM-CSF) (Peprotech/Tebu, Germany). Subsequently, BM-DCs were incubated with different concentrations of ZK191784 (10, 100 and $1000 \mathrm{nmol} / \mathrm{L})$ and stimulated overnight with $1 \mu \mathrm{g} / \mathrm{mL}$ LPS (Sigma, Germany). Supernatants were harvested for ELISA and BM-DCs were used for fluorescence-activated cell sorting (FACS) analysis.

For isolation of CD11 ${ }^{+}$DCs, spleen and MLNs were excised from ZK191784- or vehicle-treated animals $(100 \mu \mathrm{g} / \mathrm{kg}$ per day orally, for $5 \mathrm{~d})$, injected with $100 \mathrm{U} / \mathrm{mL}$ collagenase IV in RPMI (Worthington Biochemicals, Lakewood, NJ, USA) and incubated for $30 \mathrm{~min}$ at $37^{\circ} \mathrm{C}$. After incubation, organs were mechanically dissociated. In the case of the spleen, red blood cells were removed by ACK lysis. CD $11 \mathrm{c}^{+}$DCs were positively selected from single-cell suspensions from the spleen and MLNs using CD11c microbeads and a magnetic cell separation column, according to the manufacturer's instructions (Miltenyi Biotec, Bergisch Gladbach, Germany). The resulting DC preparations were $>95 \%$ CD11c-positive. Isolated DCs were stimulated overnight with $5 \mu \mathrm{g} / \mathrm{mL}$ phosphothioate-stabilized CpG-ODN with the following sequence: ODN1668 5'-TCCATGACGTTCCTGATG CT-3' (Metabion, Martinsried, Germany). Supernatants were harvested for ELISA and cells were used for FACS analysis.

\section{FACS analysis and immunohistochemistry}

Cells were analyzed by FACS using two-color staining. Briefly, isolated lymphocytes were incubated with $20 \mu \mathrm{g} / \mathrm{mL}$ of anti-CD16/CD32 mAb and 10\% FCS to block Fc receptors, and stained with both fluorescein isothiocyanate (FITC)- and phycoerythrin (PE)-conjugated mAbs. The cells were washed and analyzed by flow cytometry using an EPICS-XL MCL Coulter. For immunohistochemistry, intestinal tissue samples were snap-frozen in liquid nitrogen embedded in optimal cutting temperature medium and 5 - to $10-\mu \mathrm{m}$ cryostat sections were cut. Incubation with primary antibodies was followed by incubation with biotinylated polyclonal anti-rat IgG or anti-hamster IgG (both Dianova, Germany) mAbs as secondary antibodies. Tissue was stained using the avidin/biotin complex immunoperoxidase kit according to the manufacturer's instructions (Vector Laboratories) and developed with 3-amino-9-ethylcarbazol. Sections were counterstained with hematoxylin/eosin.

\section{Statistical analysis}

Statistical analysis was performed using the Mann-Whitney $U$ test for unpaired samples (histological score, cytokine levels) and Student's $t$ test (cytokine levels of primary DCs and BM-DCs). Differences were considered statistically significant at $P<0.05$ (labeled). For graphical analysis of the data, box plots were used. The box stretches from the lower hinge to the upper hinge and contains the middle half of the scores in the distribution. The median is shown as a line across the box, and the largest value below the upper hinge and the smallest value above the lower inner fence are drawn additionally, indicating the distribution.

\section{RESULTS}

\section{Treatment with ZK191784 ameliorates acute and chronic DSS-induced colitis}

As shown previously, vitamin $\mathrm{D}$ deficiency leads to severe colitis in IL-10 knockout mice ${ }^{[7]}$. However, limitations are involved in the study of cytokine-deficient animals, as intestinal inflammation in these mice may not represent a normal immune response, due to the lack of important regulatory cytokines. Therefore, we decided to investigate the effects of ZK191784 in the DSS models of acute and chronic colitis, as results in both models are not impaired by cytokine deficiency.

Administration of ZK191784 to mice before and during induction of acute colitis ameliorated colitis, as demonstrated by a significant reduction in the histological score by $25 \%$ compared to control mice (Figure $1 \mathrm{~A}$ : control, $7.2 \pm 0.44$ vs ZK191784, $5.4 \pm 1$ 34; $P<0.05)$, and reflected by a less severe inflammatory infiltrate and reduced epithelial damage, as seen in histological sections of the intestine (Figure 1C). Whether animals were pretreated with the calcitriol analog before induction of colitis or whether application of ZK191784 was started at the time of colitis induction made no difference in the ameliorating effect of the compound. Also, when animals were treated sequentially with the calcitriol analog during induction of chronic colitis, intestinal inflammation improved as well by $27.1 \%$ (Figure 1B: control, 7. $2 \pm 1$. 2 vs ZK191784, $5.25 \pm$ 1. 9; $P<0.05)$. As demonstrated previously $^{[26]}$, the calcitriol compound did not increase serum levels of calcium in a significant manner, as compared to serum calcium levels in control animals (data not shown). 

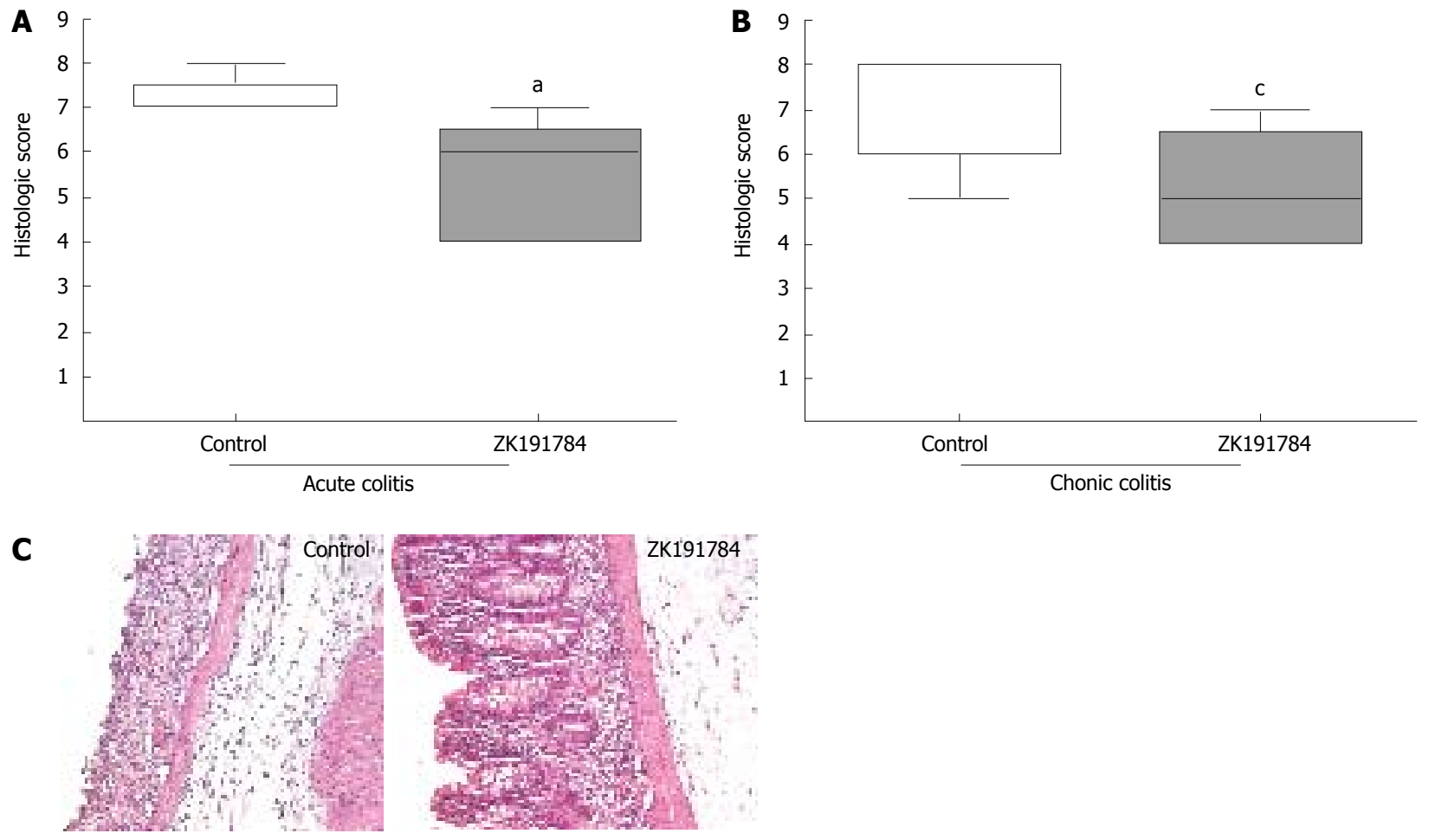

Figure 1 Treatment with ZK191784 ameliorates intestinal inflammation in acute and chronic DSS colitis. A: Acute DSS colitis was induced by administration of $3 \%$ DSS for $7 \mathrm{~d}$. ${ }^{a} P<0.05, n=5$ mice per group. Data presented are representative of three independent experiments; B: Administration of ZK191785 was started after the first and the third cycle of DSS administration for induction of chronic colitis. ${ }^{c} P<0.05, n=8$ or 9 mice per group. Data show one of two independent experiments. Data are displayed as box plots in which the box contains the middle half of the scores in the distribution, the median is shown as a line across the box, and the largest value below the upper hinge and the smallest value above the lower inner fence are indicate the distribution; C: Representative colonic hematoxylin/eosin sections from animals with acute DSS colitis treated with ZK191784 or vehicle $(\times 100)$.

\section{Treatment with ZK191784 suppresses the secretion of proinflammatory cytokines by MLN cells}

In order to further characterize the differences in colitis severity between ZK191784-treated and control animals, we compared the cytokine secretion in both groups. Therefore, MLNs of diseased animals were harvested at the end of the experiment, and levels of pro- and antiinflammatory cytokines within the supernatant of MLN cells were measured. The secretion of the proinflammatory cytokines IFN- $\gamma$ (Figure 2A) and IL-6 (data not shown) by isolated cells was dramatically suppressed by treatment with ZK191784 in acute colitis compared with control mice (IFN- $\gamma,-62.7 \%$; IL-6, $-47.4 \%, P<0.05$ ), whereas IL-10 secretion increased significantly by 2.3 -fold (Figure 2B). Similarly, in chronic colitis, spontaneous IFN- $\gamma$ secretion was suppressed by ZK191784 (control, $46.5 \pm 8.4 \mathrm{pg} /$ $\mathrm{mL}$; ZK191784, $4.2 \pm 1.2 \mathrm{pg} / \mathrm{mL}, P<0.05)$. However, in chronic colitis we were not able to detect a significant difference in the secretion of IL-6 or IL-10 by MLN cells derived from ZK191784- or vehicle-treated animals (data not shown).

Th1 cytokines play a proinflammatory role in chronic DSS-induced colitis ${ }^{[29]}$. Therefore, we evaluated the effects of ZK191784 administration on expression of the Th1-specific transcription factor T-beta in the colonic tissue, using real-time PCR. As shown, the levels of T-beta expression were significantly reduced to $18 \%$ compared with those in the control group $(P<0.0001$, Figure 2C). Additionally, even if we were not able to detect a significant difference in the secretion of the anti- inflammatory cytokine IL-10, the mRNA-levels of these cytokines increased slightly by 1.4-fold in ZK191784treated animals $(P=0.00025$, Figure $2 \mathrm{D})$.

\section{Treatment with ZK191784 prevents infiltration of intestinal lamina propria with activated $C D 11 C^{+} D C$ in animals with acute colitis}

Vitamin D3 is known to interact with antigen-presenting cells and to prevent their activation ${ }^{[30]}$, therefore, we were interested to see whether administration of the calcitriol compound ZK191784 influenced the activation of intestinal DCs within the inflamed colonic mucosa. As shown in Figure 3, immunohistochemical analysis for CD11 ${ }^{+}$DCs within the colonic lamina propria revealed a dramatic decrease in cell numbers in intestinal tissue derived from mice treated with ZK191784, as compared to that from control mice. Additionally, when staining for the activation marker $\mathrm{CD} 80$ was performed, almost no positive cells were found in the colonic mucosa of calcitriol-treated animals, whereas as expected, in control animals, large numbers of infiltrating $\mathrm{CD} 80^{+}$cells were detected. No changes were observed concerning the numbers of $\mathrm{CD}^{+}$ T cells (data not shown).

\section{ZK191784 inhibits the production of proinflammatory cytokines and the expression of costimulatory molecules by in vitro-derived DCs}

So far, our results indicated that the positive effects of ZK191784 could be mediated by an influence of the compound on antigen-presenting DCs. It has previously 
A
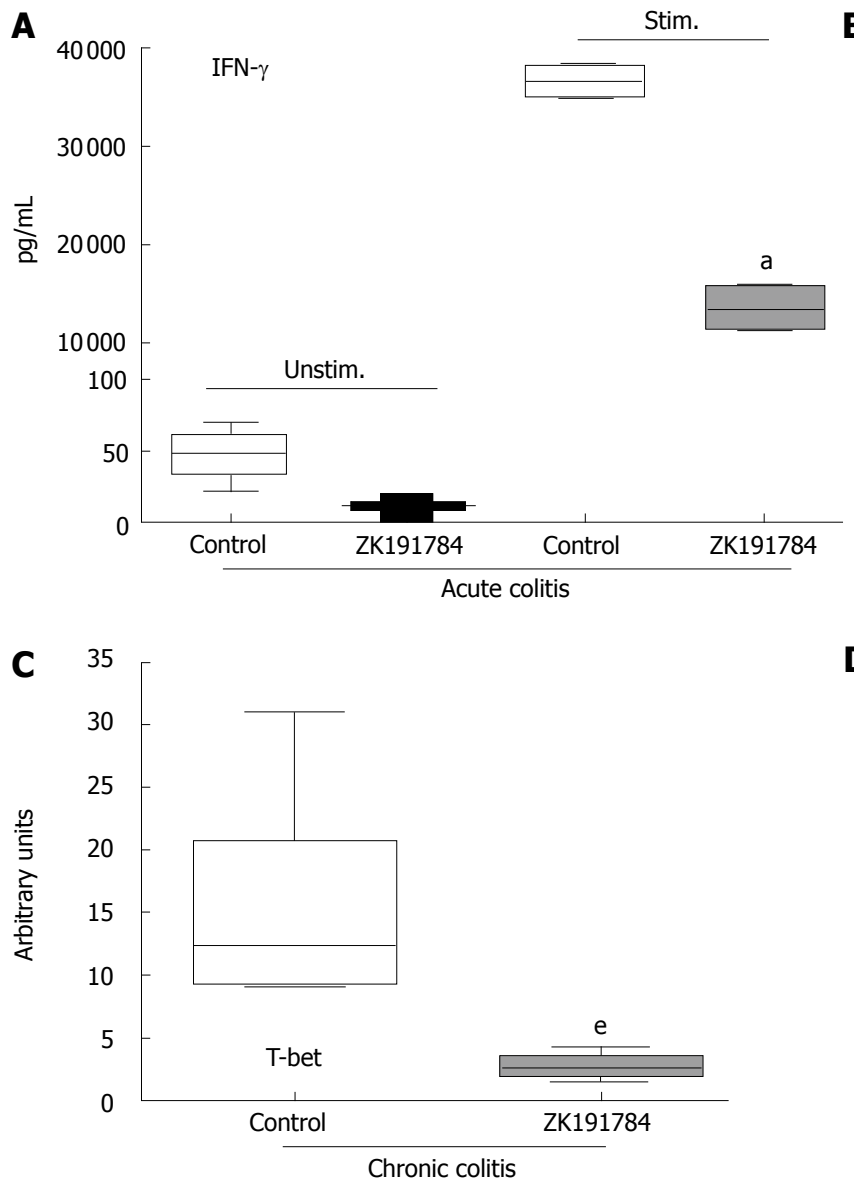

B

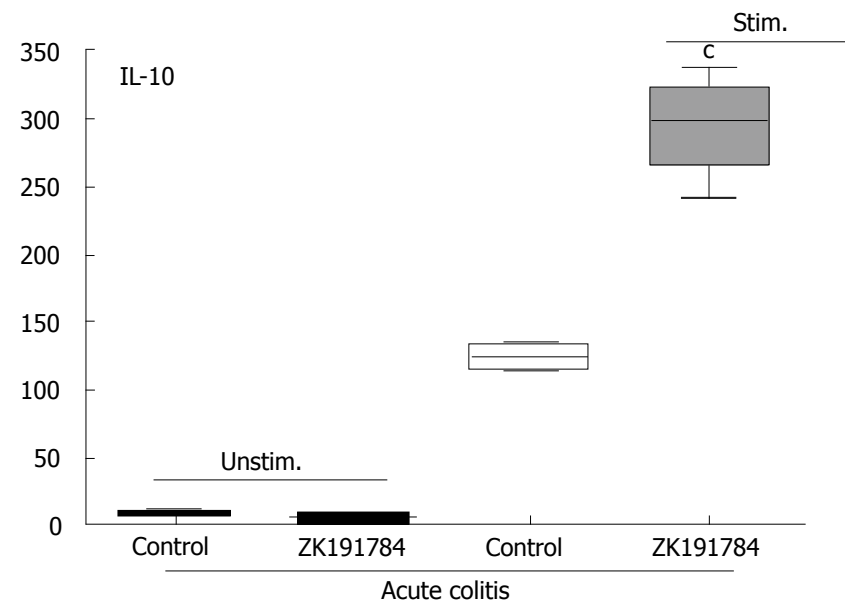

D

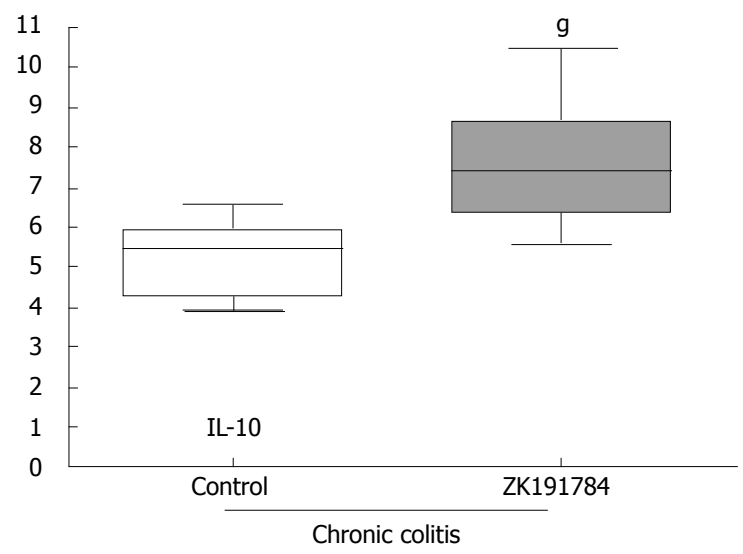

Figure 2 Effects of ZK191784 treatment on cytokine production in acute and chronic colitis. $(\mathbf{A}$ and $\mathbf{B}){ }^{\mathrm{a}} P<0.05,{ }^{\mathrm{c}} P<0.05, n=5$ animals. Data presented are representative of three independent experiments. (C and $\mathbf{D}) n=8$ or 9 animals per group. Data presented show one of two independent experiments. ${ }^{e} P<0.05,{ }^{9} P<0.05$. Data are displayed as box plots, in which the box contains the middle half of the scores in the distribution, the median is shown as a line across the box, and the largest value below the upper hinge and the smallest value above the lower inner fence indicate the distribution.

been shown that calcitriol inhibits IL-12 production by human monocytes ${ }^{[31]}$. To determine whether this capacity was shared by ZK191784, the calcitriol analog was tested for its ability to inhibit the secretion of proinflammatory cytokines by BM-DCs that were stimulated with LPS overnight in the presence of different concentrations of ZK191784.

The secretion of IL-10 was not influenced by the presence of the compound (data not shown). However, ZK191784 was able to significantly inhibit the production of the proinflammatory cytokines IFN- $\gamma$ and IL-12 by $60 \%$ and $40 \%$, respectively, in a dose-dependent manner, after stimulation of cells with LPS (Figure 4A and 4B; IFN- $\gamma, P$ $=0.0227$; IL-12, $P=0.0069)$. Additionally, FACS analysis revealed reduced expression of costimulatory molecules (CD40, CD80 and CD86) after stimulation on the surface BM-DCs exposed to ZK191784 (Figure 4C). Therefore, the presence of the calcitriol analog within cell cultures seems to inhibit the activation of in vitro-derived BM-DCs.

\section{ZK191784 inhibits the production of proinflammatory cytokines by primary DCs}

To further evaluate the effects of ZK191784 on DC in vivo, mice were treated with the calcitriol analog or vehicle, and primary $\mathrm{CD} 11 \mathrm{c}^{+} \mathrm{DCs}$ were isolated from the spleen and MLNs. FACS analysis revealed no difference in

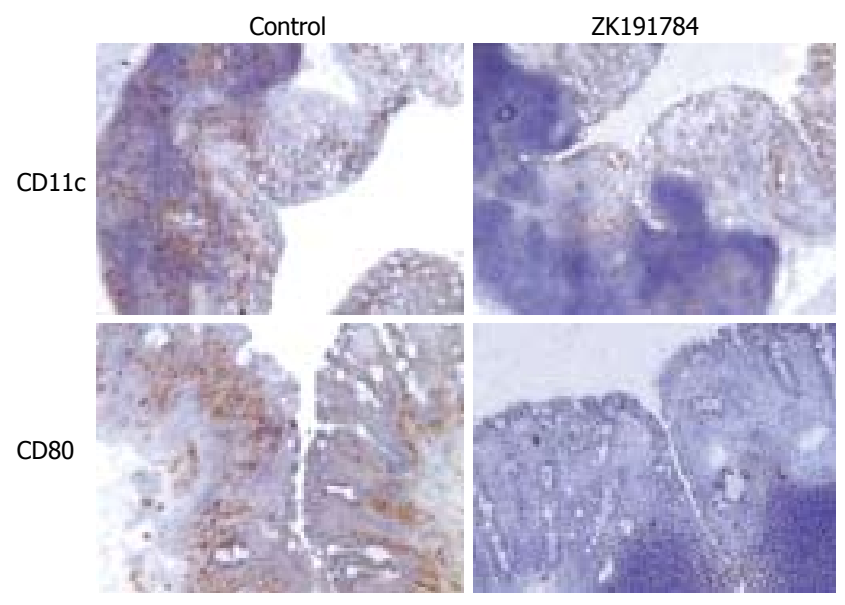

Figure 3 Effects of ZK191784 treatment on the distribution of CD11 ${ }^{+}$DCs in the colonic mucosa. Sections were stained for CD11c or the costimulatory molecule CD80 ( $\times 100)$. Staining with isotype controls revealed no background staining (data not shown). Representative sections from one of three mice per group are shown.

the expression of costimulatory molecules in DCs from different treatment groups. However, after stimulation with $\mathrm{CpG}$ in vitro, primary splenic and mesenteric DCs isolated from mice treated with ZK191784 secreted significantly lower levels of proinflammatory cytokines than those derived from control mice (Figure 5A and 5B). TNF- $\alpha$ secretion from splenic DCs from ZK191784- 
A

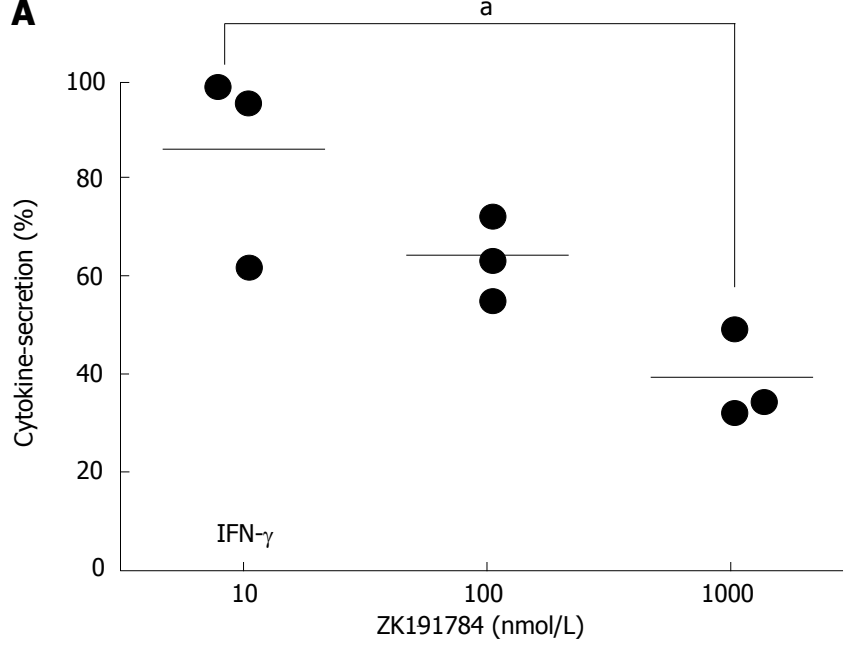

C

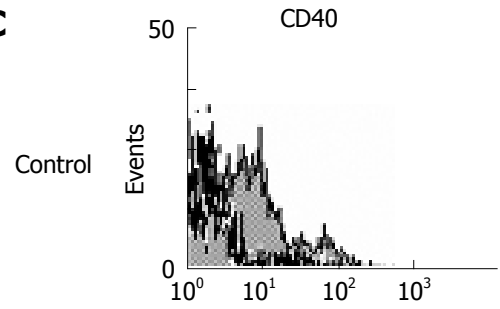

ZK191784

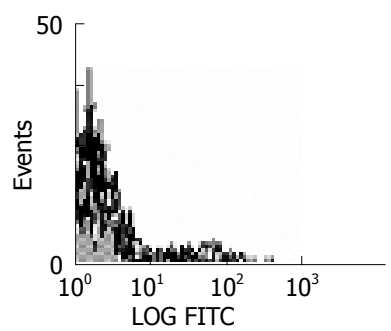

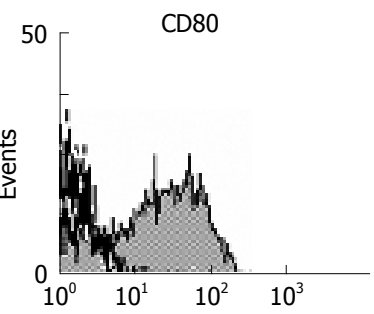

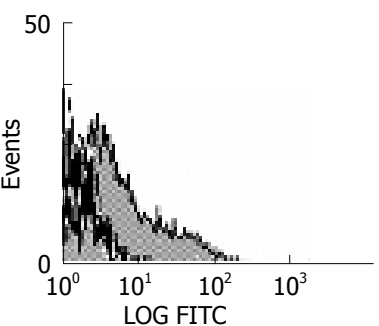

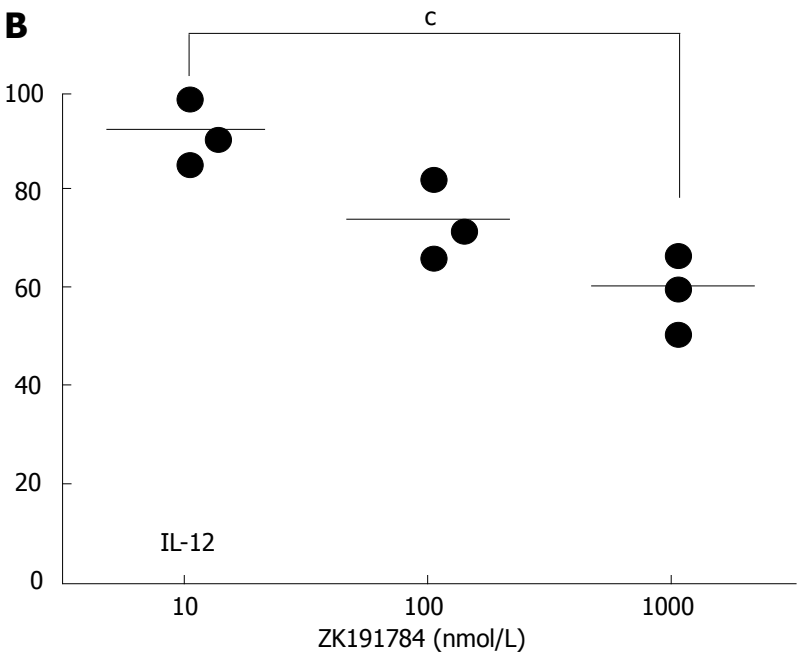
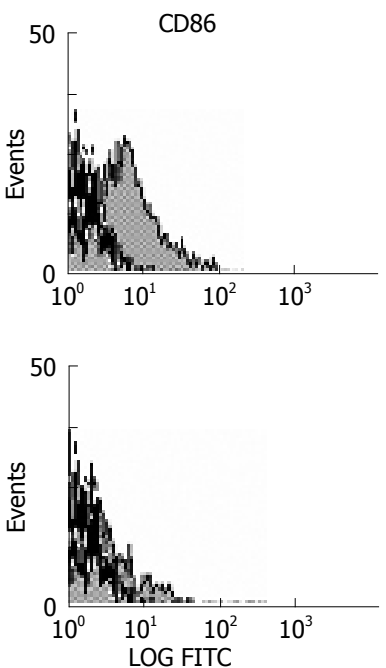

Figure 4 Effects of ZK191784 treatment on the secretion of cytokines and expression of costimulatory molecules by BM-DCs. DCs were generated from bone marrow of mice and stimulated with LPS $(1 \mu \mathrm{g} / \mathrm{mL})$ overnight in the presence of different concentrations of ZK191784 (10, $100 \mathrm{or} 1000 \mathrm{nmol} / \mathrm{L})$. Concentrations of the proinflammatory cytokines IFN- $\gamma(\mathbf{A})$ and IL-12 (B) were measured within the supernatants by ELISA. ${ }^{\mathrm{a}} P<0.05,{ }^{\mathrm{C}} P<0.05$, data presented are representative of three independent experiments. BM-DCs were cultured in the presence of ZK191784 (1000 nmol/L) or vehicle, and were stimulated overnight with LPS. CD11c ${ }^{+}$DCs were stained with FITCconjugated mAbs for expression of the costimulatory molecules CD40, CD80 and CD86. Isotype controls are shown with dark lines, and positive staining is shown in grey (C).

treated animals was reduced to $74 \%(P=0.0364)$ and IL-12 production was decreased to $62 \%$, as compared to control DCs $(P=0.0136)$. The effect was even more dramatic when secretion of proinflammatory cytokines from mucosal MLN DCs was evaluated. We were able to show that TNF- $\alpha$ and IL-12 levels from ZK191784treated mice were reduced to $9.0 \%(P=0.0005)$ and $4.4 \%$ $(P<0.0001)$, respectively, compared to cells from control animals. The results suggest that the in vivo effects of the calcitriol analog ZK191784 are more dramatic at mucosal sites of the intestine than in systemic lymphatic tissue.

\section{DISCUSSION}

In our study we extended previous data about the importance of calcitriol in colitis as we demonstrated that administration of ZK191784, a less calcemic analog of calcitriol, ameliorated not only acute but also chronic DSSinduced colitis. In contrast to previous studies, in which the influence of vitamin D deficiency was investigated in IL-10 knockout mice, our results were not obtained in cytokine-deficient animals, but in normal Balb/c mice. Additionally, our data suggested that the effects of the compound were at least partly due to its influence on DCs in the intestinal mucosa, as systemic administration of ZK191784 inhibited the activation of DCs within the spleen, MLNs and intestinal lamina propria by downmodulation of proinflammatory cytokines and inhibition of costimulatory molecule expression on the surface of DCs.

Calcitriol is known to act directly on $\mathrm{T}$ cells because vitamin D3 inhibits the proinflammatory transcription factor nuclear factor $(\mathrm{NF}) \kappa \mathrm{B}$, and subsequently impairs the expression of proinflammatory cytokines TNF- $\alpha$ and IFN- $\gamma^{20]}$. TNF- $\alpha$ is known to be an important mediator of inflammation in IBD patients ${ }^{[32]}$, and several TNF- $\alpha$ blockers are effective in $\mathrm{IBD}^{[33]}$. Therefore, any treatment that targets the TNF- $\alpha$ pathway and Th1-associated cytokines is likely to be a possible treatment for intestinal inflammation. Indeed, stimulated splenic and mesenterial DCs produced lower amounts of TNF- $\alpha$ when mice were treated with the calcitriol analog, and secretion of the 

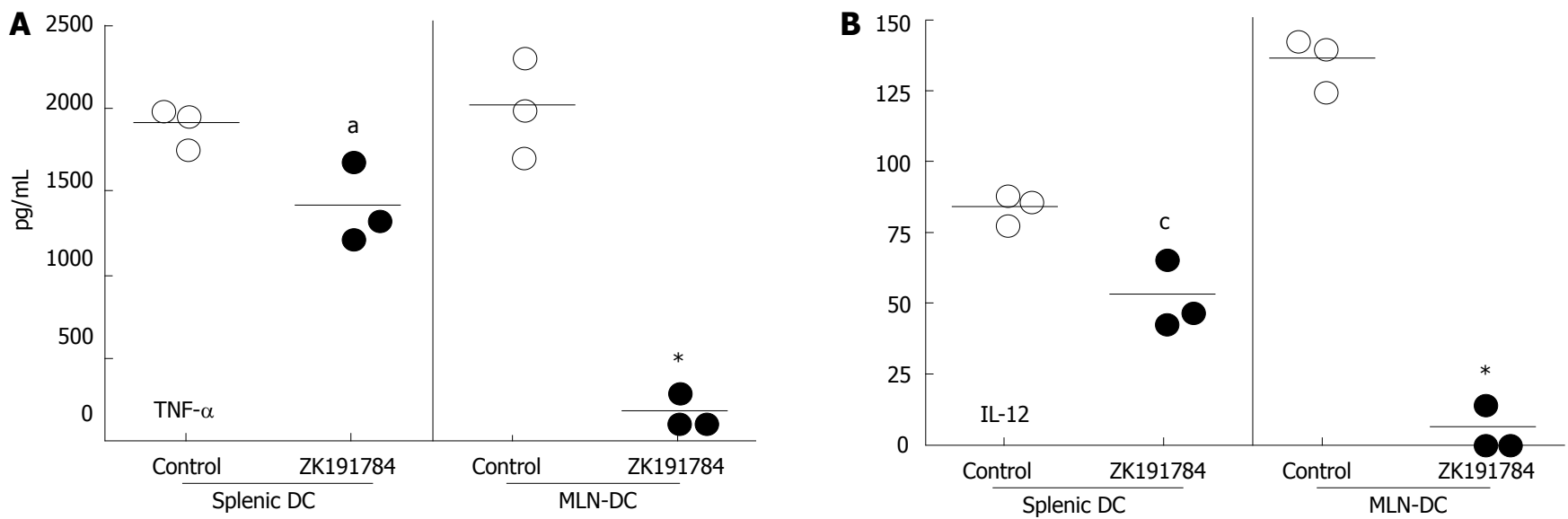

Figure 5 Effects of ZK191784 treatment on the secretion of cytokines by primary DCs derived from spleen and MLNs. CD11 ${ }^{+}$DCs were isolated from the spleen and MLNs of animals pretreated with ZK191784 or vehicle. Cells were stimulated overnight with CpG. Triplicate cultures were analyzed for each condition. Concentrations of the proinflammatory cytokines TNF- $\alpha(\mathbf{A})$ and IL-12 (B) were measured within supernatants by ELISA. ${ }^{a} P<0.05$, ${ }^{\mathrm{C}} P<0.05$, data presented are representative of three independent experiments.

proinflammatory cytokines IFN- $\gamma$ and IL- 6 by MLN cells was dramatically reduced after ZK191784 administration. On the other hand, protein levels of the anti-inflammatory cytokine IL-10 increased in acute colitis and mRNA levels of these cytokines were also enhanced in the chronic colitis model after application of ZK191784.

In vitro experiments have demonstrated that calcitriol renders DCs in a perpetual state of immaturity, as it down-regulates the expression of MHC class II and costimulatory molecules ${ }^{[34,35]}$ and decreases the capacity of myeloid human DCs to induce Th1-cell development ${ }^{[36]}$. Also, it inhibits IL-12 production by both macrophages and DCs via inhibition of $\mathrm{NF} \kappa \mathrm{B}$ activation and transcriptional repression of the IL-12p40 gene ${ }^{[31,36]}$. The proinflammatory cytokine IL-12 is involved in the pathogenesis of colitis ${ }^{[37]}$, as administration of anti-IL-12 substantially reduces the severity of intestinal inflammation, and mice treated with IL-12 during the application of DSS develop a more severe acute colitis as compared to controls ${ }^{[38]}$. Therefore, inhibition of IL-12 secretion by antigen-presenting cells can also be an important way to ameliorate disease.

However, so far, it is unclear whether mucosal DCs are also targets for the immunomodulatory activity of calcitriol. Our results demonstrate that infiltrating activated $\mathrm{CD} 11 \mathrm{c}^{+} \mathrm{DCs}$ into the intestinal lamina propria were dramatically reduced in number after systemic administration of ZK191784. Additionally, the reduced expression of costimulatory molecules by DCs, accompanied by the inhibition of proinflammatory cytokine secretion, may contribute substantially to decrease DC-dependent T-cell activation within the intestinal mucosa and could largely account for the immunosuppressive properties of this compound on acute and chronic colitis development in our model.

Interestingly, recent data have suggested that in vivo macrophages, DCs and epithelial cells are a potential source of calcitriol ${ }^{[39,40]}$. Therefore, local calcitriol production may keep antigen-presenting cells in an immature state in the healthy intestinal mucosa, and support the suppression of mucosal Th1 cytokine secretion and T-cell proliferation. This mechanism would help to establish tolerance by inhibition of unnecessary inflammatory responses. It is possible that vitamin D deficiency, either due to malnutrition or malabsorption in patients with IBD, is an additional piece of the puzzle that helps to disturb the normal immunoregulation in the gut. Our data support this hypothesis, as administration of the calcitriol analog was able to ameliorate symptoms of DSS colitis by inhibition of activation of mucosal DCs, as demonstrated by lack of expression of costimulatory molecules that would otherwise drive the inflammatory process.

Another recent study suggests an essential function for DCs in programming lymphocyte homing and microenvironmental positioning ${ }^{[41]}$. The authors were able to demonstrate that calcitriol that was processed by antigen-presenting DCs upregulated the expression of the chemokine receptor CCR10 on the surface of responding $T$ cells, and enabled them to migrate to the skin. On the other hand, the expression of the gut homing adhesion molecule $\alpha 4 \beta 7$ and the intestinal chemokine receptor CCR9 on the T-cell surface was suppressed. Therefore, it is possible that, in addition to changes in the phenotype and number of intestinal DCs, ZK191784 can act via DCs on $\mathrm{T}$ cells within the gut by up-regulation of skin homing chemokine receptors and down-modulation of gut homing molecules. This may reduce the number of infiltrating $\mathrm{T}$ cells within the gut during colitis and ameliorate severity of intestinal inflammation. Further studies are under way to investigate the expression of chemokine receptors on $\mathrm{T}$ cells in response to the calcitriol analog ZK191784.

The data presented by us and others point to a crucial role of calcitriol-regulated processes in IBD. As no interference by vitamin $\mathrm{D}$ compounds with the ability of animals to act defensively against opportunistic infections has so far been shown ${ }^{[17]}$, calcitriol may be an attractive treatment strategy compared to other immunosuppressive reagents used in IBD patients. However, so far, development of hypercalcemia limits calcitriol administration. The new calcitriol analog ZK191784 that was used in our study has previously been shown to have reduced calcemic activity and 
therefore, a more favorable therapeutic profile than calcitriol.

In conclusion, we believe that we showed for the first time that ZK191784, a low-calcemic calcitriol analog, significantly ameliorated acute and chronic DSS-induced colitis, most likely due to inhibition of DC activation that prevented development of proinflammatory pathogenic $\mathrm{T}$ cells. This less hypercalcemic calcitriol analog is therefore an attractive immunomodulatory agent with few side effects, which, either alone or in combination with other drugs, may have therapeutic applications in the treatment of IBD.

\section{COMMENTS}

\section{Background}

Chronic IBD in patients still has unknown etiology. However, it is thought that a dysregulated response of antigen-presenting DCs towards bacterial and food antigens within the gut plays a role within the disease process. Currently, treatment of disease consists of immunosuppressive drugs such as steroids and azathioprine, which have significant side effects. We investigated the effects of a calcitriol analog with few side effects for the treatment of murine colitis. Additionally, we were able to show that the calcitriol analog influenced the phenotype and number of DCs within the gut.

\section{Research frontiers}

Besides genetic factors that are thought to predispose individuals to develop $I B D$, the environment seems to contribute to the disease. There are reasons to believe that vitamin $D$ may be an environmental factor that affects colitis, since vitamin $D$ deficiency has been linked to IBD, even when the disease is in remission. Additionally, vitamin $\mathrm{D}$ deficiency has been shown to accelerate the development of colitis symptoms among mice that develop spontaneous enterocolitis. It has been shown that calcitriol selectively regulates the immune response without compromising the host's ability to fight infection, which makes it an attractive therapeutic option compared to standard immunosuppressive medication in patients with chronic colitis. However, calcitriol has clear doselimiting hypercalcemic effects that interfere with its systemic clinical use, due to a strong influence on calcium homeostasis and the risk of associated side effects.

\section{Innovations and breakthroughs}

We were able to show that treatment with ZK191784, a low-calcemic calcitriol analog, resulted in significant amelioration of murine colitis in acute and chronic intestinal inflammation. The down-regulation of colonic inflammation was associated with a dramatic reduction in the secretion of proinflammatory cytokines and a significant increase in anti-inflammatory mediators by cells within local lymph nodes. Additionally, lower numbers of infiltrating activated DCs were found in the colon in mice that were treated with the calcitriol analog, and the secretion of proinflammatory cytokines by mucosal DCs was inhibited in the presence of the calcitriol analog.

\section{Applications}

We were able to show, as far as we are aware, for the first time that a lowcalcemic calcitriol analog significantly ameliorated acute and chronic murine colitis. Inhibition of DC activation prevented development of proinflammatory pathogenic $\mathrm{T}$ cells. This calcitriol analog is therefore an attractive immunomodulatory agent with few side effects, which, either alone or in combination with other drugs, may have therapeutic applications in the treatment of IBD.

\section{Terminology}

IBD: Inflammatory bowel disease, chronic recurrent colitis in patients with an autoimmune background. DCs: Dendritic cells, cells that capture foreign antigens and present them to lymphocytes, therefore inducing an immune reaction.

\section{Peer review}

This paper by Strauch et al investigated the properties of ZK191784, a derivative analog of calcitriol (activated form of vitamin D3), with potentially fewer side effects and similar inhibition of T-cell function, to regulate colitis in an experimental mouse model. The results suggest that treatment with this inhibitor decreased the severity of acute and chronic colitis in this model.

\section{REFERENCES}

1 Bouma G, Strober W. The immunological and genetic basis of inflammatory bowel disease. Nat Rev Immunol 2003; 3: 521-533

2 Hugot JP, Chamaillard M, Zouali H, Lesage S, Cézard JP, Belaiche J, Almer S, Tysk C, O'Morain CA, Gassull M, Binder V, Finkel Y, Cortot A, Modigliani R, Laurent-Puig P, GowerRousseau C, Macry J, Colombel JF, Sahbatou M, Thomas G. Association of NOD2 leucine-rich repeat variants with susceptibility to Crohn's disease. Nature 2001; 411: 599-603

3 Sartor RB. Pathogenesis and immune mechanisms of chronic inflammatory bowel diseases. Am J Gastroenterol 1997; 92: 5S-11S

4 Andus T, Gross V. Etiology and pathophysiology of inflammatory bowel disease--environmental factors. Hepatogastroenterology 2000; 47: 29-43

5 Siffledeen JS, Siminoski K, Steinhart H, Greenberg G, Fedorak $\mathrm{RN}$. The frequency of vitamin $\mathrm{D}$ deficiency in adults with Crohn's disease. Can J Gastroenterol 2003; 17: 473-478

6 Tajika M, Matsuura A, Nakamura T, Suzuki T, Sawaki A, Kato T, Hara K, Ookubo K, Yamao K, Kato M, Muto Y. Risk factors for vitamin D deficiency in patients with Crohn's disease. J Gastroenterol 2004; 39: 527-533

7 Cantorna MT, Munsick C, Bemiss C, Mahon BD. 1,25-Dihy droxycholecalciferol prevents and ameliorates symptoms of experimental murine inflammatory bowel disease. J Nutr 2000; 130: $2648-2652$

8 Zhu Y, Mahon BD, Froicu M, Cantorna MT. Calcium and 1 alpha,25-dihydroxyvitamin D3 target the TNF-alpha pathway to suppress experimental inflammatory bowel disease. Eur J Immunol 2005; 35: 217-224

9 Daniel C, Radeke HH, Sartory NA, Zahn N, Zuegel U, Steinmeyer A, Stein J. The new low calcemic vitamin D analog 22-ene-25-oxa-vitamin D prominently ameliorates $\mathrm{T}$ helper cell type 1-mediated colitis in mice. J Pharmacol Exp Ther 2006; 319: 622-631

10 Froicu M, Weaver V, Wynn TA, McDowell MA, Welsh JE, Cantorna MT. A crucial role for the vitamin D receptor in experimental inflammatory bowel diseases. Mol Endocrinol 2003; 17: 2386-2392

11 Froicu M, Cantorna MT. Vitamin D and the vitamin D receptor are critical for control of the innate immune response to colonic injury. BMC Immunol 2007; 8: 5

12 Simmons JD, Mullighan C, Welsh KI, Jewell DP. Vitamin D receptor gene polymorphism: association with Crohn's disease susceptibility. Gut 2000; 47: 211-214

13 Bemiss CJ, Mahon BD, Henry A, Weaver V, Cantorna MT. Interleukin-2 is one of the targets of 1,25-dihydroxyvitamin D3 in the immune system. Arch Biochem Biophys 2002; 402: 249-254

14 Veldman CM, Cantorna MT, DeLuca HF. Expression of 1,25-dihydroxyvitamin $\mathrm{D}(3)$ receptor in the immune system. Arch Biochem Biophys 2000; 374: 334-338

15 Boonstra A, Barrat FJ, Crain C, Heath VL, Savelkoul HF, O'Garra A. 1alpha,25-Dihydroxyvitamin d3 has a direct effect on naive $\mathrm{CD} 4(+) \mathrm{T}$ cells to enhance the development of Th2 cells. J Immunol 2001; 167: 4974-4980

16 Cantorna MT, Humpal-Winter J, DeLuca HF. In vivo upregulation of interleukin-4 is one mechanism underlying the immunoregulatory effects of 1,25-dihydroxyvitamin $\mathrm{D}(3)$. Arch Biochem Biophys 2000; 377: 135-138

17 Cantorna MT, Hullett DA, Redaelli C, Brandt CR, HumpalWinter J, Sollinger HW, Deluca HF. 1,25-Dihydroxyvitamin D3 prolongs graft survival without compromising host resistance to infection or bone mineral density. Transplantation 1998; 66: 828-831

18 Van Etten E, Decallonne B, Verlinden L, Verstuyf A, Bouillon R, Mathieu C. Analogs of 1alpha,25-dihydroxyvitamin D3 as pluripotent immunomodulators. J Cell Biochem 2003; 88: 223-226

19 Gregori S, Giarratana N, Smiroldo S, Uskokovic M, Adorini L. A 1alpha,25-dihydroxyvitamin $\mathrm{D}(3)$ analog enhances regulatory T-cells and arrests autoimmune diabetes in NOD mice. Diabetes 2002; 51: 1367-1374

20 Stio M, Martinesi M, Bruni S, Treves C, d'Albasio G, Bagnoli S, 
Bonanomi AG. Interaction among vitamin $\mathrm{D}(3)$ analogue $\mathrm{KH}$ 1060, TNF-alpha, and vitamin D receptor protein in peripheral blood mononuclear cells of inflammatory bowel disease patients. Int Immunopharmacol 2006; 6: 1083-1092

21 Stio M, Bonanomi AG, d'Albasio G, Treves C. Suppressive effect of 1,25-dihydroxyvitamin D3 and its analogues EB 1089 and KH 1060 on T lymphocyte proliferation in active ulcerative colitis. Biochem Pharmacol 2001; 61: 365-371

22 Cantorna MT, Woodward WD, Hayes CE, DeLuca HF. 1,25-dihydroxyvitamin D3 is a positive regulator for the two anti-encephalitogenic cytokines TGF-beta 1 and IL-4. J Immunol 1998; 160: 5314-5319

23 Groux H, O'Garra A, Bigler M, Rouleau M, Antonenko S, de Vries JE, Roncarolo MG. A CD4+ T-cell subset inhibits antigenspecific T-cell responses and prevents colitis. Nature 1997; 389: 737-742

24 Penna G, Giarratana N, Amuchastegui S, Mariani R, Daniel $\mathrm{KC}$, Adorini L. Manipulating dendritic cells to induce regulatory T cells. Microbes Infect 2005; 7: 1033-1039

25 Adorini L, Penna G, Giarratana N, Roncari A, Amuchastegui $\mathrm{S}$, Daniel KC, Uskokovic M. Dendritic cells as key targets for immunomodulation by Vitamin D receptor ligands. J Steroid Biochem Mol Biol 2004; 89-90: 437-441

26 Zügel U, Steinmeyer A, Giesen C, Asadullah K. A novel immunosuppressive 1alpha,25-dihydroxyvitamin D3 analog with reduced hypercalcemic activity. I Invest Dermatol 2002; 119: $1434-1442$

27 Obermeier F, Dunger N, Strauch UG, Grunwald N, Herfarth $\mathrm{H}$, Schölmerich J, Falk W. Contrasting activity of cytosinguanosin dinucleotide oligonucleotides in mice with experimental colitis. Clin Exp Immunol 2003; 134: 217-224

28 Lutz MB, Kukutsch N, Ogilvie AL, Rössner S, Koch F, Romani N, Schuler G. An advanced culture method for generating large quantities of highly pure dendritic cells from mouse bone marrow. J Immunol Methods 1999; 223: 77-92

29 Obermeier F, Kojouharoff G, Hans W, Schölmerich J, Gross V, Falk W. Interferon-gamma (IFN-gamma)- and tumour necrosis factor (TNF)-induced nitric oxide as toxic effector molecule in chronic dextran sulphate sodium (DSS)-induced colitis in mice. Clin Exp Immunol 1999; 116: 238-245

30 Adorini L, Giarratana N, Penna G. Pharmacological induction of tolerogenic dendritic cells and regulatory T cells. Semin Immunol 2004; 16: 127-134
31 Mattner F, Smiroldo S, Galbiati F, Muller M, Di Lucia P, Poliani PL, Martino G, Panina-Bordignon P, Adorini L. Inhibition of Th1 development and treatment of chronicrelapsing experimental allergic encephalomyelitis by a nonhypercalcemic analogue of 1,25-dihydroxyvitamin D(3). Eur J Immunol 2000; 30: 498-508

32 Ganesan S, Travis SP, Ahmad T, Jazrawi R. Role of tumor necrosis factor in Crohn's disease. Curr Opin Investig Drugs 2002; 3: 1297-1300

33 Rutgeerts PJ, Targan SR. Introduction: anti-TNF strategies in the treatment of Crohn's disease. Aliment Pharmacol Ther 1999. 13 Suppl 4: 1

34 Adorini L, Penna G, Giarratana N, Uskokovic M. Tolerogenic dendritic cells induced by vitamin $\mathrm{D}$ receptor ligands enhance regulatory $\mathrm{T}$ cells inhibiting allograft rejection and autoimmune diseases. J Cell Biochem 2003; 88: 227-233

35 Griffin MD, Kumar R. Effects of 1alpha,25(OH)2D3 and its analogs on dendritic cell function. J Cell Biochem 2003; 88: 323-326

36 Penna G, Amuchastegui S, Giarratana N, Daniel KC, Vulcano M, Sozzani S, Adorini L. 1,25-Dihydroxyvitamin D3 selectively modulates tolerogenic properties in myeloid but not plasmacytoid dendritic cells. I Immunol 2007; 178: 145-153

37 Tozawa K, Hanai H, Sugimoto K, Baba S, Sugimura H, Aoshi T, Uchijima M, Nagata T, Koide Y. Evidence for the critical role of interleukin-12 but not interferon-gamma in the pathogenesis of experimental colitis in mice. J Gastroenterol Hepatol 2003; 18: 578-587

38 Kojouharoff G, Hans W, Obermeier F, Männel DN, Andus T, Schölmerich J, Gross V, Falk W. Neutralization of tumour necrosis factor (TNF) but not of IL-1 reduces inflammation in chronic dextran sulphate sodium-induced colitis in mice. Clin Exp Immunol 1997; 107: 353-358

39 Hewison M, Zehnder D, Chakraverty R, Adams JS. Vitamin $\mathrm{D}$ and barrier function: a novel role for extra-renal 1 alphahydroxylase. Mol Cell Endocrinol 2004; 215: 31-38

40 Hewison M, Gacad MA, Lemire J, Adams JS. Vitamin D as a cytokine and hematopoetic factor. Rev Endocr Metab Disord 2001; 2: 217-227

41 Sigmundsdottir H, Pan J, Debes GF, Alt C, Habtezion A, Soler D, Butcher EC. DCs metabolize sunlight-induced vitamin D3 to 'program' $\mathrm{T}$ cell attraction to the epidermal chemokine CCL27. Nat Immunol 2007; 8: 285-293

S- Editor Zhu LH L- Editor Kerr C E- Editor Lu W 